\title{
Risk Assessment in Manual Material Handling of an Edible Oil Company
}

\author{
Mohamed Ismail. S', Muthukumar. $\mathrm{K}^{2}$
}

\author{
1PG Student, 2Professor \\ 1,2Department of Mechanical Engineering, Bannari Amman Institute of Technology, \\ 1,2Sathyamangalam, Coimbatore, Tamil Nadu, India
}

\begin{abstract}
How to cite this paper: Mohamed Ismail. S | Muthukumar. K "Risk Assessment in Manual Material Handling of an Edible Oil Company" Published in International Journal of Trend in Scientific Research and Development (ijtsrd), ISSN: 24566470, Volume-3 | Issue-3, April 2019, pp.774-780, URL: https://www.ijtsrd.c om/papers/ijtsrd23 112.pdf

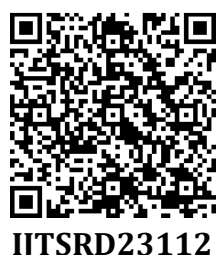

Copyright (C) 2019 by author(s) and International Journal of Trend in Scientific Research and Development Journal. This is an Open Access article distributed under the terms of the Creative Commons

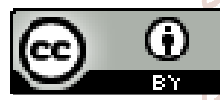
Attribution License (CC BY 4.0) (http://creativecommons.org/licenses/ by $/ 4.0$ )

\section{ABSTRACT}

The process of Shifting material manually has the potential for injury to employees. The purpose of this study is to assess and analyze the injury risk level in manual handling activity, as well as to improve the condition. The observed manual material handling activity is truck loading. This activity was analyzed using different software such as NIOSH equation, Rapid Entire Body Assessment, and compression force using 3DSSPP software as well as Nordic body map questionnaire was used to identify body discomfort level. In this study 5 male truck loading operators were taken as subjects, they are loading boxes from stacking area to truck manually. The task involved small repetitive movement while handling material, due to this the load man has faced work related musculoskeletal problems. The Nordic questionnaire is spread among the workers for collecting data, after collecting all information sorted out and analyzes the body parts using above software. The outcome shows that most of the workers who carry out activities have a high level of risk with the score of REBA exceeds points 12 (risk is very high, need immediate changes required to improve the task), Literature survey indicates the lifting index should not be in more than 1 , but the value obtained is 7.591 (the job is more dangerous and stressful). Compression force exceeds the actual limit of 3400 , but in this manual handling having compression force is 3408 . When the Nordic body mapping method the major discomfort was found in the body parts such as Lower back, Forearm, upper arm. Based on the suggestion conveyor system is introduced and we re-assess risk level of the body parts and the result shows that the risk level decreased up to $50 \%$. Therefore replacing manual handling system with the conveyor system with reduces the lower back pain.

\section{INTRODUCTION}

Manual Material Handling $(\mathrm{MMH})$ is one of the most physically difficult works in manufacturing. Back injury is one of the most frequently $(22 \%$ of all accidents that happened) problem reported in industry. One cause of this injury is occurred by over burden force, $60 \%$ of the over burden is caused by lifting and $20 \%$ pushing or pulling goods.

1. For this related manual material handling based on literature survey used to Nordic body mapping questionnaire among the workers after that collecting all information about discomfort of body parts and make it as document.

2. Study and analyze the load man job task activity and watched every sequence of motion involved in the manual material handling.

3. Taken photograph of different posture angle related MMH activity

4. After taking photos, analyzed using ergo-fellow software and the measurement feed in to software. There are different ergonomic tool used in this study such as NIOSH EQUATION, REBA, and 3DSSPP to find out COMPRESSION FORCE.

5. Suggestion and Modification needed to be applied to the load man and the posture is reassessed.
To investigate the manual material handling work system of the worker and to identify major areas causing injury of worker that associated to the activities. The study was conducted in a reputed edible oil company. The major purpose is to reduce the risk of worker physiological and physical disorders caused by poor Manual Material Handling system. Musculoskeletal Disorder (MSDs) issue is harm in muscles, nerves, ligaments, tendons, joints. Muscles wounds can be in types of muscle strain, aggravation, what's more, degeneration. In the interim, bone wounds might be types of wounding, small scale breaks, cracks, or contorted bones [1]. Body position incredibly influences the event of high hazard, particularly the back position. There are three upgrades connected to decrease the danger of damage to the movement, including improving the creation of pressing, improving body stance, and making rule notices [2]. This investigation was directed to evaluate $\mathrm{MMH}$ errands and, along these lines, the outcomes are relevant for overhauling the MMH assignments. Taking remedial activities to improve MMH assignments appears to be basic. The ergonomics mediation should concentrate on overhauling them. As per our discoveries, lifting and bringing down assignments ought to be viewed as basic and organized in taking restorative activities [3]. A numerical model for weariness amassing and 
rest remittance estimation is created. As expressed in the presentation, this model expects to be connected to manual material taking care of exercises where the entire body is included, deciding general body weakness, which is regularly estimated with vitality use rate [4]. Have attempted an alternate methodology utilizing thorough investigation of distress of various body parts. Maybe a couple of them clubbed together and giving a poll. The creators have exhibited an institutionalized poll for the examination of musculoskeletal manifestations in an ergonomic or word related wellbeing setting is displayed. The mapping of various body parts is substantially more clear and particular than the CMDQ [5]. Have investigated as far as a study of Malaysian shape making industry. Shape making industry is a maker and creator for different sorts of form, for example, ware shape, bundling mold and furniture form for other assembling forms. Because of the shape fabricating process, specialists are presented to the dangers of upper appendage issue, for example, redundant assignments, awkward work stances and carryout work for extensive stretches without break. Organized meeting utilizing Cornell Musculoskeletal Discomfort Questionnaires (CMDQ) were directed more than 35 specialists in form making industry [6]. The fundamental motivation behind this examination was to survey potential age related contrasts in trunk kinematics (i.e., proportions of work techniques) and the outcome insect mechanical requests on the lower back amid two symmetric MMH errands Five sexual orientation adjusted age bunches were shaped to empower progressively explicit assessment of age related contrasts in MMH bio mechanics. Such received kinematics brought about bearing a higher shearing load on the lower back in more established members (i.e., affirming our speculation) [7]. This technique was utilized to comprehend the inclination of distress which indicates on explicit body parts. Nine laborers who deal with stacking and accumulation narrows were identified to have LBP and MSD issue side effects. This implies the present technique utilized ought to be researched and quick changes ought to be connected. Should this technique is proceeded with, a higher danger of LBP and MSD could happen. The administration ought to underline on the structure of the work stations also. A decent plan ought to limit the necessity of MMH exercises that devours high measure of vitality, for example, the usage of transports and rails to transport crude material to the heater [8]. Distinguishing proof of word related hazard components and appraisal of those hazard factors with the assistance of biomechanical assessment for different development related MMH assignments are two basic issues for research. The proposed research system tends to these two issues just as progress approach from biomechanical point of view [9]. In view of numerous analyses they made an original informational collection and utilized it to assemble strategic relapse models to distinguish critical factors and characterize manual lifting errands into high hazard and generally safe regarding LBDs. In spite of the fact that the exhibitions of our best models are superior to those detailed in National Institute for Occupational Health and Safety (NIOHS) Guides and two of our past examinations, they are commonly less hopeful than those announced in a few different investigations; this paper proposes a progressively orderly and dependable way to deal with making and approving classifiers to recognize low and high hazard manual lifting employments that add to LBDs [10].

\subsection{Aim of the Study}

First is to do a survey and using a questionnaire (Nordic body mapping) to find out the level of discomfort faced by load man and stacking man while performing their job in different postures. This survey make the load man to look back the work related musculoskeletal disorders they have faced and different aspects related to work and their health affected by that. It also help in assessing the body parts which were especially in a vulnerable position when they work. The questionnaire technique which is distribute among the workers in each truck loader and then collecting all information of discomfort of body parts.

Secondly the study will try to look into the current misgivings in the type of postures the workers are put in using the software analysis. The postures are mimicked using the ergo-fellow and 3DSSPP software the level of discomfort is determined. Then the workers are given ergonomic guidelines and reassessing the new postures in the same way. The comparison of the results finally is aimed to show how the simple knowledge of ergonomics can make a difference in a daily life of a worker.

\section{METHODOLOGY}

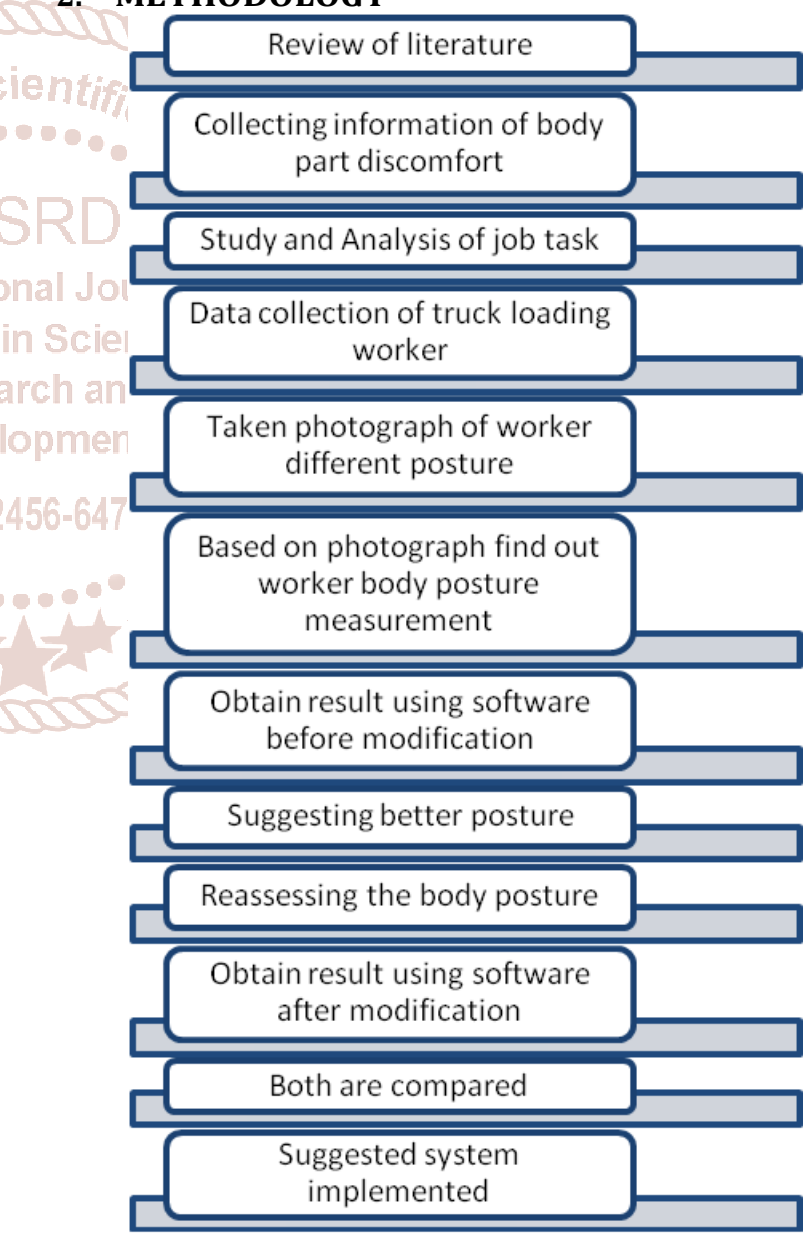

\subsection{Software were used to assess body posture Ergo-fellow}

The Ergo-Fellow programming has 17 ergonomic apparatuses to assess and improve work environments conditions, so as to diminish work related risk. The product is extremely helpful for ergonomists and for all experts in the zone of word related safety and health. The software was developed by FBF SISTEMAS in 2009 and It's also very good for educational purposes. 
International Journal of Trend in Scientific Research and Development (IJTSRD) @ www.ijtsrd.com eISSN: 2456-6470

\section{DSSPP Software}

3D SSPP programming predicts static quality necessities for assignments, for example, lifts, presses, pushes, and pulls. The program gives an inexact activity reenactment that incorporates pose information, constrain parameters and male/female anthropometry. Examination is supported by a programmed stance age highlight and three-dimensional human realistic illustrations.

\subsection{OPERATOR DATA}

A total of 5 goods loading worker were approached for the survey. The worker has loading goods manually. During manual handling a load man can lift the box having weight is $15 \mathrm{~kg}$ and working on $12 \mathrm{hr}$ per day as shown in fig 2.1. Worker details given in Table 2.1

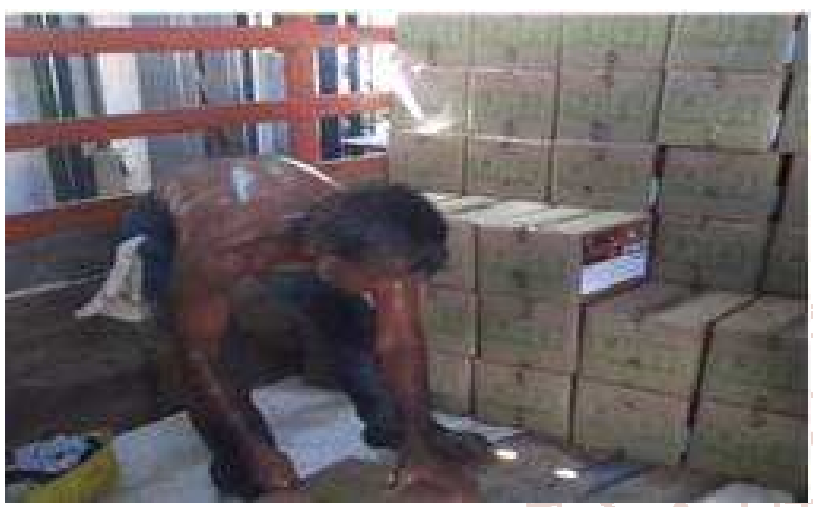

Fig: 2.1 A load man has load the box from stacking area to truck by manually

\begin{tabular}{|c|c|c|c|c|c|c|c|c|}
\hline $\begin{array}{c}\text { S1 } \\
\text { no }\end{array}$ & Name & Experience & Age & $\begin{array}{c}\text { Weight } \\
(\mathrm{kg})\end{array}$ & $\begin{array}{c}\text { Height } \\
(\mathrm{cm})\end{array}$ & $\begin{array}{c}\text { Arm } \\
\text { Length } \\
(\mathrm{cm})\end{array}$ & $\begin{array}{c}\text { Hip } \\
\text { height } \\
(\mathrm{cm})\end{array}$ & $\begin{array}{c}\text { Shoulder } \\
\text { Length } \\
(\mathrm{cm})\end{array}$ \\
\hline 1 & $\begin{array}{c}\text { Load } \\
\text { man 1 }\end{array}$ & 8 year & 38 & 68 & 167 & 55 & 88 & 38 \\
\hline 2 & $\begin{array}{c}\text { Load } \\
\text { man 2 }\end{array}$ & 3 year & 44 & 74 & 165 & 52 & 85 & 40 \\
\hline 3 & $\begin{array}{c}\text { Load } \\
\text { man 3 }\end{array}$ & 2 year & 40 & 77 & 172 & 60 & 95 & 42 \\
\hline 4 & $\begin{array}{c}\text { Load } \\
\text { man 4 }\end{array}$ & 2 year & 39 & 65 & 170 & 58 & 91 & 36 \\
\hline 5 & $\begin{array}{c}\text { Load } \\
\text { man 5 }\end{array}$ & 6 year & 35 & 69 & 161 & 55 & 84 & 34 \\
\hline
\end{tabular}

Tabled: 2.1 Worker general information

\section{FINDING THE RISK IN MANUAL HANDLING (Before Improvement of the Task)}

\subsection{NIOSH EQUATION}

A man has to load the object from stacking area to truck loading by manually. This picture fig 3.1 showed a man manually handling load with help of body force.

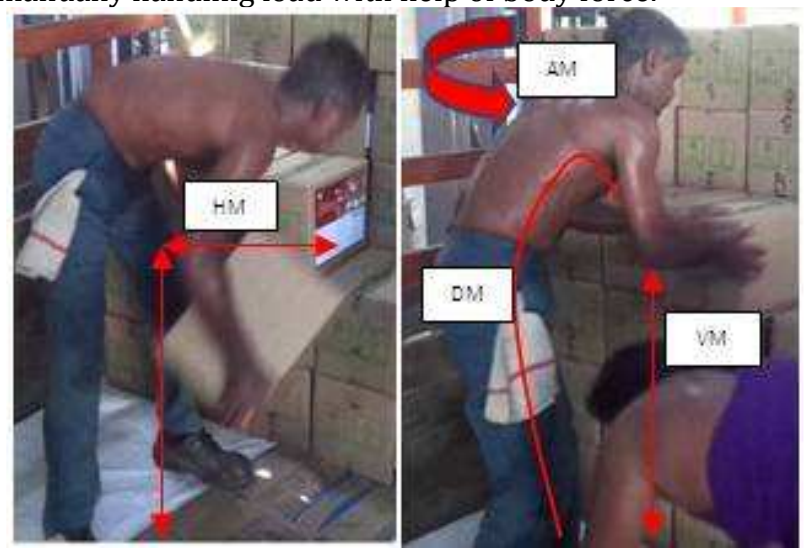

Fig: 3.1 Handling of Material Manually

\begin{tabular}{|c|c|c|c|c|c|c|c|}
\hline $\begin{array}{c}\text { LIFTING } \\
\text { EQUATION }\end{array}$ & $\begin{array}{c}\text { HM } \\
\mathbf{c m}\end{array}$ & $\begin{array}{c}\text { VM } \\
\mathbf{c m}\end{array}$ & $\begin{array}{c}\text { DM } \\
\mathbf{C m}\end{array}$ & $\begin{array}{c}\text { AM } \\
\text { degree }\end{array}$ & $\begin{array}{c}\text { FM } \\
\text { Lift/min }\end{array}$ & $\begin{array}{c}\text { CM } \\
\text { LOAD } \\
\text { Kg }\end{array}$ \\
\hline LOAD MAN 1 & 37 & 75 & 75 & 83 & $14 \mathrm{box} / \mathrm{min}$ & 0.95 & 15 \\
\hline LOAD MAN 2 & 40 & 75 & 75 & 78 & $12 \mathrm{box} / \mathrm{min}$ & 0.95 & 15 \\
\hline LOAD MAN 3 & 39 & 75 & 75 & 81 & $13 \mathrm{box} / \mathrm{min}$ & 0.95 & 15 \\
\hline LOAD MAN 4 & 36 & 75 & 75 & 76 & $12 \mathrm{box} / \mathrm{min}$ & 0.95 & 15 \\
\hline LOAD MAN 5 & 38 & 75 & 75 & 82 & $12 \mathrm{box} / \mathrm{min}$ & 0.95 & 15 \\
\hline AVG & $\mathbf{3 8}$ & $\mathbf{7 5}$ & $\mathbf{7 5}$ & $\mathbf{8 0}$ & $\begin{array}{c}\mathbf{1 2} \\
\text { box/min }\end{array}$ & $\begin{array}{c}\text { Fair } \\
\mathbf{0 . 9 5}\end{array}$ & $\mathbf{1 5}$ \\
\hline
\end{tabular}

Table: 3.1 Load man software data before modification

According to lifting equation Collecting data from different load man then take an average value to make data collection work sheet (table 3.2)

\begin{tabular}{|c|c|c|c|c|c|c|c|}
\hline & \multicolumn{7}{|c|}{ NIOSH LIFTING VARIABLES } \\
\hline $\begin{array}{c}\text { Lifting } \\
\text { Task }\end{array}$ & $\begin{array}{c}\mathrm{H} \\
\text { Horizont } \\
\mathrm{al} \\
\text { location } \\
\mathrm{cm}\end{array}$ & $\begin{array}{c}\mathrm{V} \\
\text { Vertical } \\
\text { location } \\
\mathrm{cm}\end{array}$ & $\begin{array}{c}\mathrm{D} \\
\text { Distance } \\
\text { travel } \\
\mathrm{cm}\end{array}$ & $\begin{array}{c}\mathrm{A} \\
\text { Angle of } \\
\text { Asymmetr } \\
\mathrm{y}\end{array}$ & $\begin{array}{c}\text { F } \\
\text { Frequenc } \\
\text { degree }\end{array}$ & $\begin{array}{c}\mathrm{C} \\
\text { Couplin } \\
\mathrm{g}\end{array}$ & $\begin{array}{c}\text { Load } \\
\text { Lifte } \\
\mathrm{d}\end{array}$ \\
$\begin{array}{c}\text { Truck } \\
\text { loading } \\
\text { activity }\end{array}$ & 38 & 75 & 75 & 80 & $\begin{array}{c}12 \mathrm{box} / \mathrm{mi} \\
\mathrm{n} \\
\mathrm{kg} \\
(0.21)\end{array}$ & $\begin{array}{c}\text { Fair } \\
0.95\end{array}$ & 15 \\
\hline
\end{tabular}

Table: 3.2 NIOSH data collection worksheet for load man

The horizontal distance at the destination of the lift is $38 \mathrm{~cm}$, primarily The altitude of base is $0 \mathrm{~cm}$ and destination altitude is $75 \mathrm{~cm}$, the coupling is distinct as "fair" (i.e., $\mathrm{C}=0.95)$. Asymmetric lifting movement is concerned $(\mathrm{A}=80$ degree). Since the lifting pattern has been doing 90 minute, the lifting frequency is $12 \mathrm{boxes} / \mathrm{min}(\mathrm{F}=0.21)$. The object of load is $15 \mathrm{~kg}$, finally RWL obtained from software after that using lifting index equation (Weight $\div$ RWL $=$ LI) find out value.

\section{Hazard Assessment}
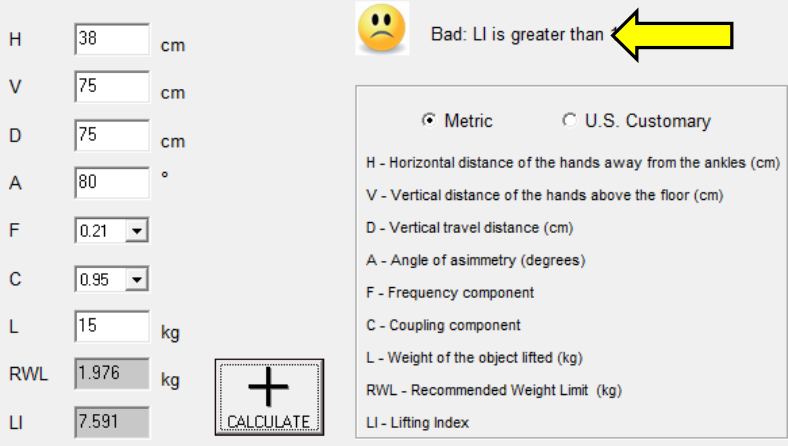

Fig: 3.2 Result of NIOSH Equation Lifting Index

The Recommended Weight Lifting for this activity is 1.976 at the destination. If we divide the weight to be lifted (15) to Recommended Weight limit (RWL=1.976), we got LI (Lift Index) value ( $\mathrm{LI}=7.591)$.Therefore $\mathrm{LI}$ is greater than 1 , These values indicate that a man doing the job more dangerous and stressful, in future a man face longtime chronic disease.

\subsection{Rapid Entire Body Analysis (REBA)}

This ergonomic appraisal tool is utilizes in a methodical procedure to assess the load man entire body postural MSD and dangers related with occupation job tasks. Score with Level of MSD risk are shown in fig 3.3. 
International Journal of Trend in Scientific Research and Development (IJTSRD) @ www.ijtsrd.com eISSN: 2456-6470

\begin{tabular}{|c|l|}
\hline Score & Level of MSD Risk \\
\hline 1 & negligible risk, no action required \\
\hline $2-3$ & low risk, change may be needed \\
\hline $4-7$ & medium risk, further investigation, change soon \\
\hline $8-10$ & high risk, investigate and implement change \\
\hline $11+$ & very high risk, implement change \\
\hline
\end{tabular}

Fig: 3.3 level of MSD risk

Collecting data and prepared chart from load man as shown in table 3.3. The load man's body posture angle average value was taken and entered in the ergo-fellow software; finally the REBA score is obtained.

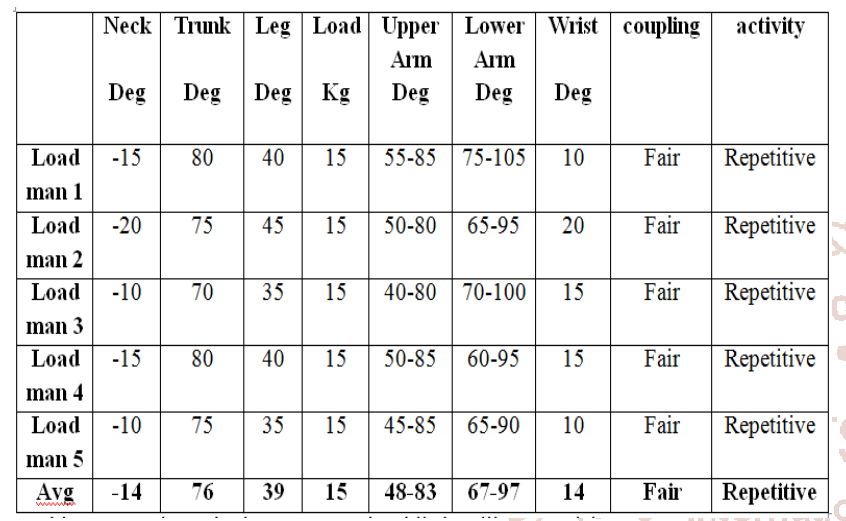

Table: 3.3 Load man body posture angle while handling material

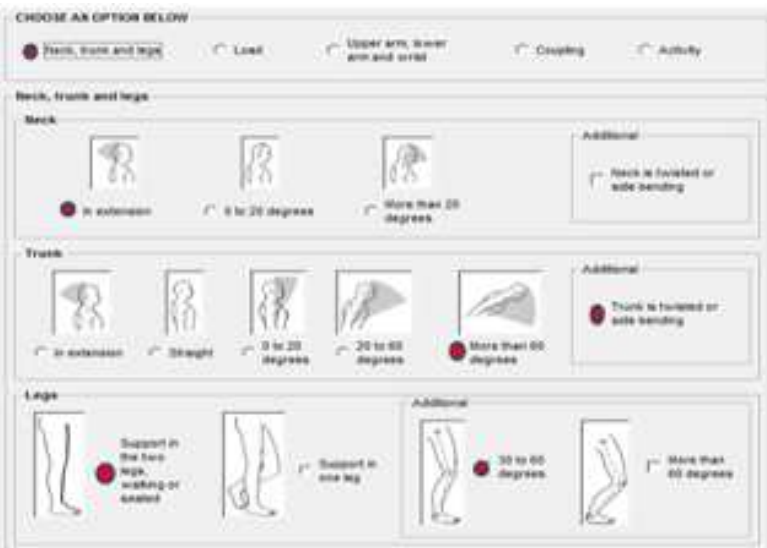

Fig: 3.4 Neck, Trunk, leg posture angle in Ergo-fellow software

Similarly each factors (Load, Upper arm, Lower arm, Wrist) of REBA Risk analysis angle entered into software and obtained risk level of REBA

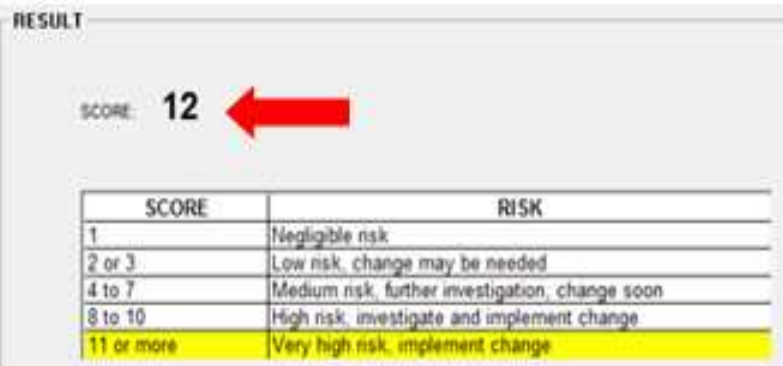

Fig: 3.5 REBA score in Ergo-Fellow software

\section{REBA Result}

Research and

The score of the REBA (11 or more) shows the risk was very high and needed immediate changes to improve the task or engineering control.

\begin{tabular}{|c|c|c|c|c|c|c|c|c|c|c|c|c|c|c|c|c|c|c|c|c|c|c|}
\hline & \multicolumn{3}{|c|}{ NECK } & \multicolumn{3}{|c|}{ SHOULDER } & \multicolumn{3}{|c|}{ UPPER BACK } & \multicolumn{3}{|c|}{ UPPER ARM } & \multicolumn{3}{|c|}{ Lower back } & \multicolumn{3}{|c|}{ Fore arm } & \multicolumn{3}{|c|}{ wrist } \\
\hline & & 1hr & $4 \mathrm{hr}$ & 8 her & 1hr & 4hr & 8hr & 1hr & 4hr & 8hit & 1hr & 4hr & 8hr & 1hr & $4 h r$ & 8hr & 1hr & $4 h r$ & ghr & 1hr & 4hr & 8hr \\
\hline \multirow{2}{*}{$\begin{array}{l}\text { IOAD } \\
\text { Man } 1\end{array}$} & $R$ & \multirow[t]{2}{*}{2} & \multirow[t]{2}{*}{2} & \multirow[t]{2}{*}{2} & 2 & 2 & 2 & \multirow[t]{2}{*}{3} & \multirow[t]{2}{*}{3} & \multirow[t]{2}{*}{2} & 3 & 3 & 3 & \multirow[t]{2}{*}{3} & \multirow[t]{2}{*}{3} & \multirow[t]{2}{*}{3} & 2 & 2 & 3 & 2 & 2 & 3 \\
\hline & $\mathrm{L}$ & & & & 2 & 2 & 2 & & & & 3 & 3 & 3 & & & & 2 & 2 & 3 & 2 & 2 & 3 \\
\hline \multirow{2}{*}{$\begin{array}{l}\text { LOAD } \\
\text { MAN } 2\end{array}$} & $R$ & \multirow[t]{2}{*}{1} & \multirow[t]{2}{*}{2} & \multirow[t]{2}{*}{2} & 2 & 2 & 2 & \multirow[t]{2}{*}{1} & \multirow[t]{2}{*}{2} & \multirow[t]{2}{*}{1} & 2 & 3 & 3 & \multirow[t]{2}{*}{3} & \multirow[t]{2}{*}{3} & \multirow[t]{2}{*}{3} & 2 & 3 & 3 & 3 & 3 & 2 \\
\hline & $\mathrm{L}$ & & & & 2 & 2 & 2 & & & & 2 & 3 & 3 & & & & 2 & 3 & 3 & 3 & 3 & 2 \\
\hline \multirow{2}{*}{$\begin{array}{l}\text { LOAD } \\
\text { MAN } 3\end{array}$} & $R$ & \multirow[t]{2}{*}{2} & \multirow[t]{2}{*}{2} & \multirow[t]{2}{*}{3} & 2 & 2 & 2 & 2 & 3 & 3 & 1 & 2 & 2 & 3 & 3 & 3 & 2 & 3 & 3 & 3 & 3 & 2 \\
\hline & $\mathrm{L}$ & & & & 2 & 2 & 2 & & & & 1 & 2 & 2 & & & & 2 & 3 & 3 & 3 & 3 & 2 \\
\hline LOAD & $\mathbf{R}$ & 2 & 2 & 2 & 2 & 3 & 2 & 1 & 1 & 1 & 3 & 3 & 3 & 3 & 3 & 3 & 3 & 3 & 3 & 2 & 3 & 3 \\
\hline MAN 4 & $\mathrm{t}$ & & & & 2 & 3 & 2 & & & & 3 & 3 & 3 & & & & 3 & 3 & 3 & 2 & 3 & 3 \\
\hline LOAD & $R$ & 1 & 2 & 2 & 1 & 2 & 2 & 1 & 1 & 1 & 2 & 3 & 3 & 3 & 3 & 3 & 2 & 3 & 3 & 2 & 2 & 2 \\
\hline MAN S & L & & & & 1 & 2 & 2 & & & & 2 & 3 & 3 & & & & 2 & 3 & 3 & 2 & 2 & 2 \\
\hline AVG & & 1.6 & 2 & 2.2 & 1.8 & 2.2 & 2 & 1.6 & 2 & 1.6 & 2.2 & 2.8 & 2.8 & 3 & 3 & 3 & 2.2 & 2.8 & 3 & 2.4 & 2.6 & 2.4 \\
\hline
\end{tabular}

\begin{tabular}{|c|c|c|c|c|c|c|c|c|c|c|c|c|c|}
\hline & \multicolumn{3}{|l|}{ HIP } & \multicolumn{3}{|c|}{ THIGH } & \multicolumn{3}{|c|}{ KNEE } & \multicolumn{3}{|c|}{ IOWER IEG } \\
\hline & & 1hr & 4 hr & 8hr & 1hr & 4 hr & ght & 1he & 4ht & 8he & 1hr & $4 \mathrm{hr}$ & $8 \mathrm{ht}$ \\
\hline \multirow{2}{*}{$\begin{array}{l}\text { LOAD } \\
\text { MAN } 1\end{array}$} & \multirow{2}{*}{\multicolumn{2}{|c|}{1}} & \multirow[t]{2}{*}{1} & \multirow[t]{2}{*}{1} & 3 & 3 & 3 & 3 & 3 & 3 & 3 & 3 & 3 \\
\hline & L & & & & 3 & 3 & 3 & 3 & 3 & 3 & 3 & 3 & 3 \\
\hline \multirow{2}{*}{$\begin{array}{l}\text { LOAD } \\
\text { MAN } 2\end{array}$} & $\mathbf{R}$ & \multirow[t]{2}{*}{1} & \multirow[t]{2}{*}{1} & \multirow[t]{2}{*}{1} & 2 & 2 & 2 & 2 & 3 & 3 & 2 & 2 & 2 \\
\hline & L & & & & 2 & 2 & 2 & 2 & 3 & 3 & 2 & 2 & 2 \\
\hline \multirow{2}{*}{$\begin{array}{l}\text { LOAD } \\
\text { MAN } 3\end{array}$} & $\mathbf{R}$ & \multirow[t]{2}{*}{1} & \multirow[t]{2}{*}{1} & \multirow[t]{2}{*}{1} & 2 & 2 & 3 & 2 & 3 & 3 & 2 & 2 & 3 \\
\hline & L. & & & & 2 & 2 & 3 & 2 & 3 & 3 & 2 & 2 & 3 \\
\hline \multirow{2}{*}{$\begin{array}{l}\text { LOAD } \\
\text { MAN } 4\end{array}$} & $\mathbf{R}$ & \multirow[t]{2}{*}{1} & \multirow[t]{2}{*}{1} & \multirow[t]{2}{*}{1} & 2 & 2 & 2 & 3 & 3 & 3 & 3 & 3 & 3 \\
\hline & L & & & & 2 & 2 & 2 & 3 & 3 & 3 & 3 & 3 & 3 \\
\hline \multirow{2}{*}{$\begin{array}{l}\text { LOAD } \\
\text { MAN S }\end{array}$} & $\mathbf{R}$ & \multirow[t]{2}{*}{1} & \multirow[t]{2}{*}{1} & \multirow[t]{2}{*}{1} & 1 & 2 & 2 & 2 & 2 & 3 & 1 & 2 & 2 \\
\hline & $\mathbf{L}$ & & & & 1 & 2 & 2 & 2 & 2 & 3 & 1 & 2 & 2 \\
\hline AVG & & 1 & 1 & 1 & 2 & 2.2 & 2.4 & 2.4 & 2.8 & 3 & 2.2 & 2.8 & 3 \\
\hline
\end{tabular}

\section{1- Slightly uncomfortable \\ 2- Moderately uncomfortable \\ 3. Very uncomfortable}

Table: 3.4 Load man discomfort frequency of Nordic body mapping using questionnaire method before modification. 


\subsection{Nordic Body Mapping}

Nordic body mapping survey was used to recognize symptoms, complaints, disorder and injuries faced by worker. This is for identification of body parts of the personnel repeatedly receiving the discomfort complaints.

The Nordic body map survey is distributed among the employees in each truck loader and collected information to make table of Discomfort frequency as shown in table 3.4

\subsubsection{Discomfort Analysis}

Following activity were made for ergonomic analysis of the truck loading worker. The checklist results showed that the major discomfort was found in the body parts are:

\section{Upper Arm \\ Lower Back \\ Forearm \\ Wrist \\ Knee}

\subsection{Compression Force}

The product of 3D SSPP is utilized to discover the most extreme power endured by the laborer in doing some kind of task. the compression force is still inside as far as possible or under $3400 \mathrm{~N}$, at that point it very well may be reasoned that the most extreme power that must be acknowledged is as yet adequate for the labors body. The truck loader posture designed in 3DSSPP software as shown in fig 3.6.

\begin{tabular}{|c|c|c|c|c|c|c|c|c|}
\hline & \multirow[t]{2}{*}{ Sex } & \multirow{2}{*}{$\begin{array}{c}\text { Feet } \\
\text { Support }\end{array}$} & \multirow{2}{*}{$\begin{array}{c}\text { Body } \\
\text { posture }\end{array}$} & \multirow{2}{*}{$\begin{array}{c}\text { Height } \\
\text { Cm }\end{array}$} & \multirow{2}{*}{$\begin{array}{c}\text { Weight } \\
\text { Cm }\end{array}$} & \multicolumn{2}{|c|}{ Hand load (N) } & \multirow{2}{*}{$\begin{array}{c}\text { Type of } \\
\text { load } \\
\text { Handling }\end{array}$} \\
\hline & & & & & & Right & Left & \\
\hline $\begin{array}{c}\text { Load } \\
\text { Man } 1\end{array}$ & Male & $\begin{array}{l}\text { Both } \\
\text { Feet }\end{array}$ & Bending & 68 & 167 & 73 & 73 & Lifting \\
\hline $\begin{array}{c}\text { Load } \\
\text { Man } 2 \\
\end{array}$ & Male & $\begin{array}{l}\text { Both } \\
\text { Feet }\end{array}$ & Bending & 74 & 165 & 73 & 73 & Lifting \\
\hline $\begin{array}{c}\text { Load } \\
\text { Man } 3 \\
\end{array}$ & Male & $\begin{array}{l}\text { Both } \\
\text { Feet }\end{array}$ & Bending & 77 & 172 & 73 & 73 & Lifting \\
\hline $\begin{array}{c}\text { Load } \\
\text { Man } 4 \\
\end{array}$ & Male & $\begin{array}{l}\text { Both } \\
\text { Feet }\end{array}$ & Bending & 65 & 170 & 73 & 73 & Lifting \\
\hline $\begin{array}{c}\text { Load } \\
\text { Man } 5 \\
\end{array}$ & Male & $\begin{array}{l}\text { Both } \\
\text { Feet }\end{array}$ & Bending & 69 & 161 & 73 & 73 & Lifting \\
\hline Avg & Male & $\begin{array}{l}\text { Both } \\
\text { Feet }\end{array}$ & Bending & 70 & 167 & 73 & 73 & Lifting \\
\hline
\end{tabular}

Table: 3.5 Data collection for compression force before modification

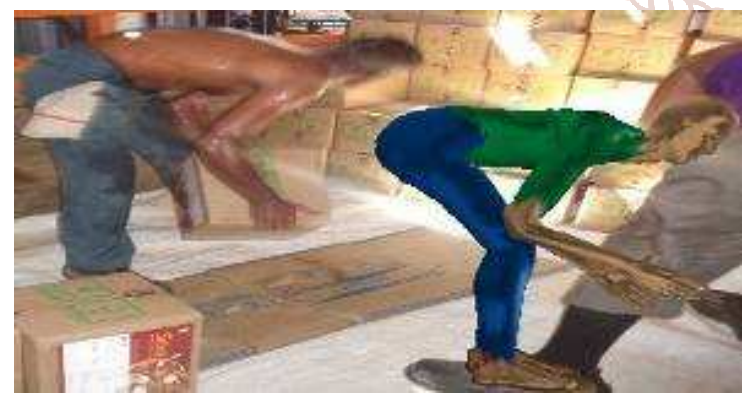

Fig: 3.6 Make material handling Anatomy posture in 3DSSPP software

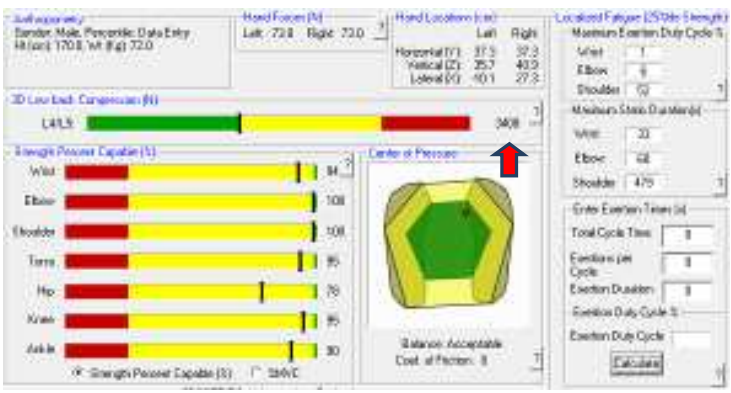

Fig: 3.7 Result of Compression Force acting on body
A worker wants to take object and load to the truck. The load man was doing job continuously at this awkward position, resulting in a high score.

\section{RESULT AND DISSCUSSION Improvement of the Task)}

\subsection{NIOSH EQUATION}

A load man batches the boxes to truck by conveyor system showing fig 4.1

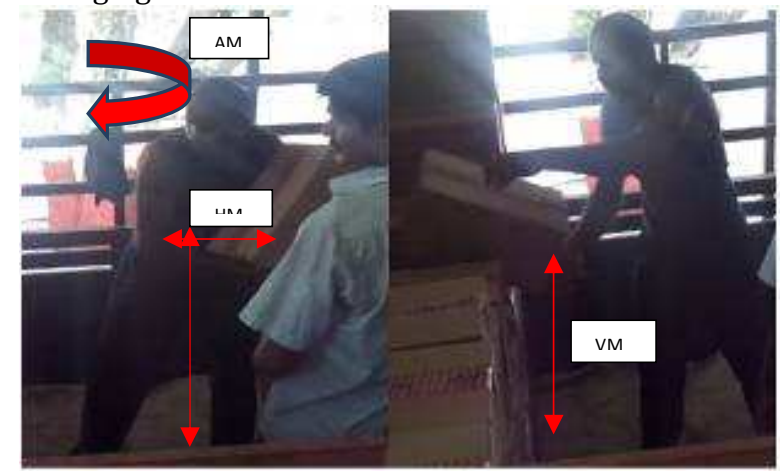

Fig: 4.1 Handling of material manually using conveyor system

\begin{tabular}{|c|c|c|c|c|c|c|c|}
\hline $\begin{array}{c}\text { LIFTING } \\
\text { EQUATION }\end{array}$ & $\begin{array}{c}\text { HM } \\
\mathbf{c m}\end{array}$ & $\begin{array}{c}\text { VM } \\
\mathbf{c m}\end{array}$ & $\begin{array}{c}\text { DM } \\
\mathbf{C m}\end{array}$ & $\begin{array}{c}\text { AM } \\
\text { degree }\end{array}$ & $\begin{array}{c}\text { FM } \\
\text { Lift/min }\end{array}$ & $\begin{array}{c}\text { CM } \\
\text { LOAD } \\
\text { Kg }\end{array}$ \\
\hline LOAD MAN 1 & 26 & 75 & 4 & 74 & $12 \mathrm{box} / \mathrm{min}$ & 1 & 15 \\
\hline LOAD MAN 2 & 31 & 75 & 3 & 77 & $10 \mathrm{box} / \mathrm{min}$ & 1 & 15 \\
\hline LOAD MAN 3 & 27 & 75 & 4 & 73 & $8 \mathrm{box} / \mathrm{min}$ & 1 & 15 \\
\hline LOAD MAN 4 & 29 & 75 & 5 & 77 & $9 \mathrm{box} / \mathrm{min}$ & 1 & 15 \\
\hline LOAD MAN 5 & 27 & 75 & 4 & 74 & $11 \mathrm{box} / \mathrm{min}$ & 1 & 15 \\
\hline AVG & $\mathbf{2 8}$ & $\mathbf{7 5}$ & $\mathbf{4}$ & $\mathbf{7 5}$ & $\begin{array}{c}\mathbf{1 0} \\
\text { box } / \mathrm{min}\end{array}$ & $\begin{array}{c}\text { Good } \\
\mathbf{( 1 )}\end{array}$ & $\mathbf{1 5}$ \\
\hline
\end{tabular}

Table: 4.1 load man data collection after modification

\begin{tabular}{|c|c|c|c|c|c|c|c|}
\hline & \multicolumn{7}{|c|}{ NIOSH LIFTING VARIABLES } \\
\hline $\begin{array}{c}\text { Lifting } \\
\text { Task }\end{array}$ & $\begin{array}{c}\text { H } \\
\text { Horizontal } \\
\text { location } \\
\mathrm{cm}\end{array}$ & $\begin{array}{c}\text { V } \\
\text { Vertical } \\
\text { location } \\
\mathrm{cm}\end{array}$ & $\begin{array}{c}\text { D } \\
\text { Distance } \\
\text { travel } \\
\mathrm{cm}\end{array}$ & $\begin{array}{c}\text { A } \\
\text { Angle of } \\
\text { Asymmetry } \\
\text { degree }\end{array}$ & $\begin{array}{c}\text { F } \\
\text { Frequency } \\
\text { Lift/min }\end{array}$ & $\begin{array}{c}\text { C } \\
\text { Coupling }\end{array}$ & $\begin{array}{c}\text { Load } \\
\text { Lifted } \\
\mathrm{kg}\end{array}$ \\
\hline $\begin{array}{c}\text { Truck } \\
\text { loading } \\
\text { activity }\end{array}$ & 28 & 75 & 4 & 75 & $\begin{array}{c}10 / \mathrm{min} \\
(0.26)\end{array}$ & $\begin{array}{c}\text { good } \\
(1)\end{array}$ & 15 \\
\hline
\end{tabular}

Table: 4.2 NIOSH data collection worksheet after modification

The horizontal distance at the target of the lift is $28 \mathrm{~cm}$. primarily the altitude of product base level is $0 \mathrm{~cm}$ and destination altitude is $75 \mathrm{~cm}$ from ground. The coupling is definite as "good" ( $\mathrm{C}=1)$. Asymmetric lifting is concerned (A $=75$ degree). Since the lifting pattern has been doing 90 minute, the lifting frequency is 10 boxes $/ \mathrm{min}(\mathrm{F}=0.26)$.

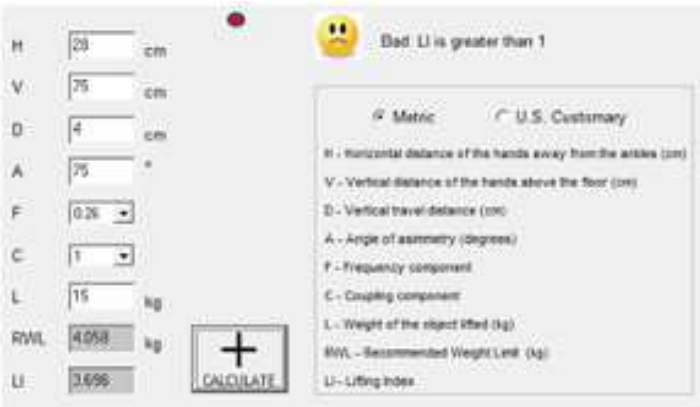

Fig:4.2 Result of NIOSH Equation Lifting Index after modification 
International Journal of Trend in Scientific Research and Development (IJTSRD) @ www.ijtsrd.com eISSN: 2456-6470

The Recommended Weigh Lifting for this activity is 4.058 at the destination. If we divide the weight to be lifted (15) to Recommended Weight limit (RWL=4.058), we got LI (Lift Index) value ( $\mathrm{LI}=3.696)$. Therefore $\mathrm{LI}$ is greater than 1 , these values indicate that a man doing the job more dangerous and stressful, at the same time after implementing new task LI value reduced up to $50 \%$ compared to existing system.

\subsection{REBA (Rapid Entire Body Analysis)}

REBA risk analysis done for load man after implement recommendation and Load man posture angle value taken using software and make it as table is shown in table 4.3

\begin{tabular}{|c|c|c|c|c|c|c|c|c|c|}
\hline & Neck & Trunk & Leg & Load & $\begin{array}{c}\text { Upper } \\
\text { Arm } \\
\text { Deg }\end{array}$ & $\begin{array}{c}\text { Lower } \\
\text { Arm } \\
\text { Deg }\end{array}$ & Wrist & Doupling & activity \\
\hline $\begin{array}{c}\text { Load } \\
\text { man 1 }\end{array}$ & 21 & 17 & 28 & 15 & 23 & 112 & 12 & Good & Repetitive \\
\hline $\begin{array}{c}\text { Load } \\
\text { man 2 }\end{array}$ & 19 & 19 & 21 & 15 & 27 & 108 & 15 & Good & Repetitive \\
\hline $\begin{array}{c}\text { Load } \\
\text { man 3 }\end{array}$ & 22 & 16 & 24 & 15 & 28 & 106 & 22 & Good & Repetitive \\
\hline $\begin{array}{c}\text { Load } \\
\text { man 4 }\end{array}$ & 20 & 19 & 24 & 15 & 31 & 110 & 9 & Good & Repetitive \\
\hline $\begin{array}{c}\text { Load } \\
\text { man 5 }\end{array}$ & 23 & 18 & 27 & 15 & 29 & 115 & 13 & Good & Repetitive \\
\hline Avg & $\mathbf{2 1}$ & $\mathbf{1 7 . 8}$ & $\mathbf{2 4 . 8}$ & $\mathbf{1 5}$ & $\mathbf{2 7 . 6}$ & $\mathbf{1 1 0 . 2}$ & $\mathbf{1 4 . 2}$ & Good & Repetitive \\
\hline
\end{tabular}

Table: 4.3 Load man body posture angle after modification

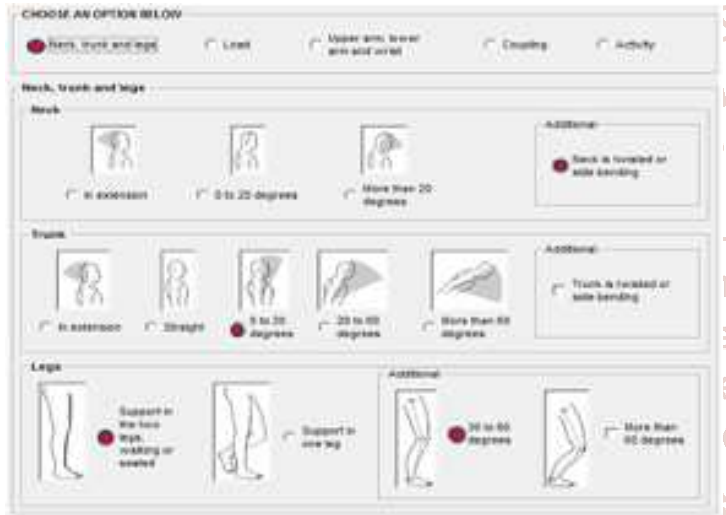

Fig: 4.3 Neck, Trunk, leg posture angle in Ergo-fellow software
SCORE:

\begin{tabular}{|l|l|}
\hline \multicolumn{1}{|c|}{ SCORE } & \multicolumn{1}{c}{ RISK } \\
\hline 1 & Negligible risk \\
\hline 2 or 3 & Low risk, change may be neeced \\
\hline 4 to 7 & Medium risk, turther imestigation, change soon \\
\hline 8 to 10 & High isk, investigate and implement change \\
\hline 11 of more & Very high risk, implement change \\
\hline
\end{tabular}

Fig: 4.4 REBA score after modification

\section{REBA Result}

After implementing new system REBA score decreased upto $50 \%$ compared to existing system. The score of the REBA 6, therefore the risk was MIDIUM, needed further investigation to improve the task soon or engineering control.

\subsection{Nordic Body Mapping}

Similarly using Nordic body mapping questionnaire, collecting data based on load man discomfort feel after implementing suggestion make it table 4.4 .

\subsubsection{DISCOMFROT ANALYSIS}

Therefore Following activity was made for ergonomic analysis of the Truck loading worker. The Checklist results showed that the Major discomfort was found in the body parts are:

\section{Lower back (lumber)} Knee

\begin{tabular}{|c|c|c|c|c|c|c|c|c|c|c|c|c|c|c|c|c|c|c|c|c|c|c|}
\hline & & \multicolumn{3}{|c|}{ NECK } & \multicolumn{3}{|c|}{ SHOULDER } & \multicolumn{3}{|c|}{ UPPER BACK } & \multicolumn{3}{|c|}{ UPPER ARM } & \multicolumn{3}{|c|}{ Lower back } & \multicolumn{3}{|c|}{ Fore arm } & \multicolumn{3}{|c|}{ wrist } \\
\hline & & 1hr & 4he & $8 \mathrm{hr}$ & 1hir & $4 \mathrm{hr}$ & $8 \mathrm{hr}$ & the & $4 h r$ & $8 \mathrm{hr}$ & 1hr & the & 8 he & thr & $4 \mathrm{hr}$ & $8 \mathrm{hr}$ & 1hr & 4he & 8hir & thr & Ahr & $8 \mathrm{hr}$ \\
\hline \multirow{2}{*}{$\begin{array}{l}\text { LOAD } \\
\text { Man } 1\end{array}$} & \multirow{2}{*}{\multicolumn{2}{|c|}{$\begin{array}{ll}\mathrm{R} & 1 \\
\mathrm{n} & \end{array}$}} & \multirow[t]{2}{*}{1} & \multirow[t]{2}{*}{2} & 1 & 2 & 2 & \multirow[t]{2}{*}{1} & \multirow[t]{2}{*}{1} & \multirow[t]{2}{*}{1} & 2 & 2 & 2 & \multirow[t]{2}{*}{2} & \multirow[t]{2}{*}{2} & \multirow[t]{2}{*}{2} & 2 & 2 & 2 & 1 & 2 & 2 \\
\hline & & & & & 1 & 2 & 2 & & & & 2 & 2 & 2 & & & & 2 & 2 & 2 & 1 & 2 & 2 \\
\hline \multirow{2}{*}{$\begin{array}{l}\text { LOAD } \\
\text { MAN } 2\end{array}$} & $R$ & \multirow[t]{2}{*}{1} & \multirow[t]{2}{*}{1} & \multirow[t]{2}{*}{1} & 1 & 1 & 2 & \multirow[t]{2}{*}{1} & \multirow[t]{2}{*}{1} & \multirow[t]{2}{*}{1} & 1 & 2 & 2 & \multirow[t]{2}{*}{2} & \multirow[t]{2}{*}{2} & \multirow[t]{2}{*}{1} & 1 & 1 & 2 & 1 & 2 & 2 \\
\hline & $\mathrm{L}$ & & & & 1 & 1 & 2 & & & & 1 & 2 & 2 & & & & 1 & 1 & 2 & 1 & 2 & 2 \\
\hline \multirow{2}{*}{$\begin{array}{l}\text { LOAD } \\
\text { MAN } 3\end{array}$} & $R$ & \multirow[t]{2}{*}{0} & \multirow[t]{2}{*}{0} & \multirow[t]{2}{*}{1} & 1 & 1 & 1 & 0 & 0 & 0 & 1 & 2 & 2 & 1 & 2 & 1 & 1 & 1 & 1 & 1 & 1 & 1 \\
\hline & $\mathrm{L}$ & & & & 1 & 1 & 1 & & & & 1 & 2 & 2 & & & & 1 & 1 & 1 & 1 & 1 & 1 \\
\hline LOAD & $\mathrm{R}$ & 0 & 0 & 1 & 0 & 0 & 1 & 0 & 1 & 0 & 1 & 1 & 1 & 1 & 2 & 1 & 1 & 2 & 2 & 1 & 1 & 1 \\
\hline MAN 4 & 1 & & & & 0 & 0 & 1 & & & & 1 & 1 & 1 & & & & 1 & 2 & 2 & 1 & 1 & 1 \\
\hline LOAD & $R$ & 0 & 0 & 0 & 0 & 0 & 1 & 0 & 0 & 0 & 1 & 1 & 1 & 1 & 1 & 1 & 1 & 1 & 2 & 1 & 1 & 1 \\
\hline MAN 5 & $\mathrm{~L}$ & & & & 0 & 0 & 1 & & & & 1 & 1 & 1 & & & & 1 & 1 & 2 & 1 & 1 & 1 \\
\hline AVG & & 0.4 & 0.4 & 1 & 0.6 & 0.8 & 1.4 & 0.4 & 0.6 & 0.4 & 1.2 & 1.6 & 1.6 & 1,4 & 1.8 & 1.2 & 1.2 & 1.4 & 1.8 & 1 & 1.4 & 1,4 \\
\hline
\end{tabular}

\begin{tabular}{|c|c|c|c|c|c|c|c|c|c|c|c|c|c|}
\hline & \multicolumn{3}{|l|}{ HIP } & \multicolumn{3}{|c|}{ THIGH } & \multicolumn{3}{|c|}{ KNEE } & \multicolumn{3}{|c|}{ LOWER LEG } \\
\hline & & the & $4 \mathrm{hr}$ & 8 he & thr & the & $8 \mathrm{hr}$ & the & $4 \mathrm{hr}$ & 8 hit & the & 4 hr & $8 \mathrm{hr}$ \\
\hline \multirow{2}{*}{$\begin{array}{l}\text { LOAD } \\
\text { MAN } 1\end{array}$} & $\mathrm{R}$ & \multirow[t]{2}{*}{1} & \multirow[t]{2}{*}{1} & \multirow[t]{2}{*}{1} & 1 & 2 & 2 & 2 & 2 & 2 & 2 & 2 & 2 \\
\hline & $\mathrm{t}$ & & & & 1 & 2 & 2 & 2 & 2 & 2 & 2 & 2 & 2 \\
\hline \multirow{2}{*}{$\begin{array}{l}\text { LOAD } \\
\text { MAN } 2\end{array}$} & $R$ & \multirow[t]{2}{*}{1} & \multirow[t]{2}{*}{1} & \multirow[t]{2}{*}{1} & 1 & 1 & 1 & 2 & 2 & 1 & 1 & 1 & 2 \\
\hline & $\mathrm{L}$ & & & & 1 & 1 & 1 & 2 & 2 & 1 & 1 & 1 & 2 \\
\hline \multirow{2}{*}{$\begin{array}{l}\text { LOAD } \\
\text { MAN } 3\end{array}$} & $R$ & \multirow[t]{2}{*}{0} & \multirow[t]{2}{*}{0} & \multirow[t]{2}{*}{0} & 1 & 1 & 1 & 1 & 2 & 2 & 1 & 1 & 1 \\
\hline & $\mathrm{t}$ & & & & 1 & 1 & 1 & 1 & 2 & 2 & 1 & 1 & 1 \\
\hline \multirow{2}{*}{$\begin{array}{l}\text { LOAD } \\
\text { MAN } 4\end{array}$} & $\mathrm{R}$ & \multirow[t]{2}{*}{0} & \multirow[t]{2}{*}{0} & \multirow[t]{2}{*}{0} & 1 & 1 & 0 & 1 & 2 & 1 & 1 & 1 & 1 \\
\hline & L & & & & 1 & 1 & 0 & 1 & 2 & 1 & 1 & 1 & 1 \\
\hline \multirow{2}{*}{$\begin{array}{l}\text { LOAD } \\
\text { MAN S }\end{array}$} & $R$ & \multirow[t]{2}{*}{0} & \multirow[t]{2}{*}{0} & \multirow[t]{2}{*}{0} & 0 & 1 & 1 & 1 & 2 & 2 & 0 & 1 & 2 \\
\hline & $\mathrm{t}$ & & & & 0 & 1 & 1 & 1 & 2 & 2 & 0 & 1 & 2 \\
\hline AVG & & 0.4 & 0.4 & 0.4 & 0.8 & 1.2 & 1 & 1.4 & 2 & 1.6 & 1 & 1.2 & 1.6 \\
\hline
\end{tabular}

1. Slightly uncomfortable

2. Moderately uncomfortable

3. Very uncomfortable

Fig: 4.4 Load man discomfort frequency of Nordic Body mapping using questionnaire method after modification 


\subsection{Compression Force}

The product of 3D SSPP is utilized to discover the most extreme power endured by the laborer in doing some kind of stress full task. The anatomy posture as shown in fig 4.5

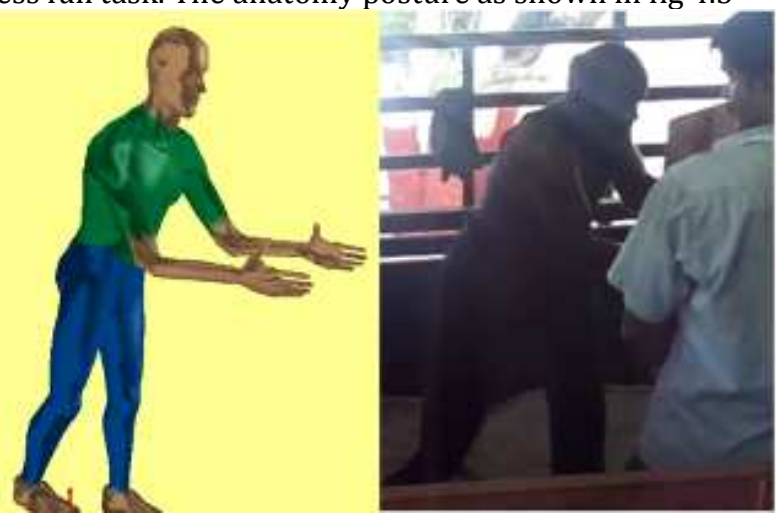

Fig: 4.5 Making material handling Anatomy posture after modification in 3DSSPP software

\begin{tabular}{|c|c|c|c|c|c|c|c|c|}
\hline & \multirow[t]{2}{*}{$5 e x$} & \multirow{2}{*}{$\begin{array}{c}\text { Fet } \\
\text { Suppont }\end{array}$} & \multirow{2}{*}{$\begin{array}{l}\text { Body } \\
\text { pesture }\end{array}$} & \multirow{2}{*}{$\begin{array}{l}\text { Heizht } \\
\mathrm{Cm}\end{array}$} & \multirow{2}{*}{$\begin{array}{l}\text { Weight } \\
\mathrm{Cm}\end{array}$} & \multicolumn{2}{|c|}{ Hand load (N) } & \multirow{2}{*}{$\begin{array}{l}\text { Type of } \\
\text { boad } \\
\text { Hasdlieg }\end{array}$} \\
\hline & & & & & & Right & Left & \\
\hline $\begin{array}{l}\text { Load } \\
\text { Man } 1\end{array}$ & Male & $\begin{array}{l}\text { Both } \\
\text { Feet }\end{array}$ & Standing & 68 & 167 & 73 & 73 & Lifting \\
\hline $\begin{array}{c}\text { Load } \\
\text { Man } 2\end{array}$ & Male & $\begin{array}{l}\text { Both } \\
\text { Feet }\end{array}$ & Srapding & 74 & 165 & 73 & 73 & Liffing \\
\hline $\begin{array}{c}\text { Load } \\
\operatorname{Man} 3\end{array}$ & Male. & $\begin{array}{l}\text { Botb } \\
\text { Feet }\end{array}$ & Standing & 77 & 172 & 73 & 73 & Lifting \\
\hline $\begin{array}{c}\text { Load } \\
\text { Man } 4\end{array}$ & Male & $\begin{array}{l}\text { Beth } \\
\text { Feel }\end{array}$ & Standing & 65 & 170 & 73 & 73 & Linigg \\
\hline $\begin{array}{c}\text { Load } \\
\text { Man } 5\end{array}$ & Male & $\begin{array}{l}\text { Borlh } \\
\text { Feer }\end{array}$ & Standing : & 69 & 161 & 73 & 73 & Linisg \\
\hline$A v g$ & Male & $\begin{array}{l}\text { Both } \\
\text { Feet }\end{array}$ & Standing & 70 & 167 & 73 & 73 & L.tring \\
\hline
\end{tabular}

Table: 4.6 Data collection for compression force after modification

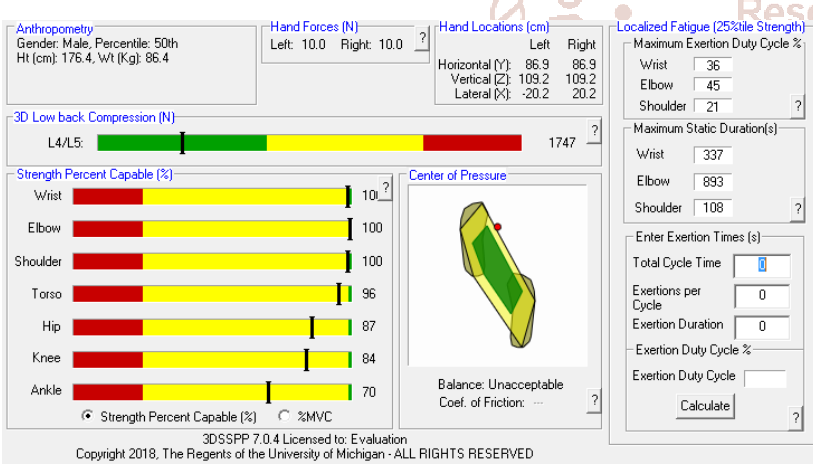

Fig: 4.6 Result of Compression Force acting on the body after modification

\subsection{Comparison Table:}

\begin{tabular}{|c|c|c|c|c|c|c|}
\hline & \multicolumn{2}{|c|}{ NIOSH - LI value } & \multicolumn{2}{|c|}{ REBA score } & \multicolumn{2}{|c|}{ Compression Force (N) } \\
\hline Criteria & Current & proposed & Current & proposed & Current & Proposed \\
\hline $\begin{array}{l}\text { Truck } \\
\text { Loading }\end{array}$ & $7.591>1$ & $3.696>1$ & $\begin{array}{l}12 \\
\text { High } \\
\text { Risk }\end{array}$ & \begin{tabular}{|l}
6 \\
Medium \\
Risk
\end{tabular} & 3408 & 1747 \\
\hline
\end{tabular}

Table: 4.7 Comparison Table (Before and After)

\section{CONCLUSION}

Using NIOSH, REBA, and Compression force acting on the body and Nordic body mapping, these are the Manual handling activity recognize that approximately all activities have high-risk levels. Body position very much affects the occurrence of MSD, particularly the personal back position. When we applying improvements to reduce risk of body injury like Train the worker to correct lifting techniques, improving body posture, and making guideline posters. Final conclusion is above the process we can't eliminate fully manual material handling $(\mathrm{MMH})$ activity. If replacing mechanical material handling or automatic material handling is only possible solution of this activity, but we can't replace fully automated machine because cost of process installation is very high. So best way to deal with the problem is to modify or improve the task. In this study reduced manual handling risk was reduced up to $50 \%$ from existing process.

\section{Reference}

[1] Setyanto, Nasir Widha, et al. "Ergonomics Analysis in the Scarfing Process by OWAS, NIOSH and Nordic Body Map's Method at Slab Steel Plant's Division." Ergonomics 4.3 (2015).

[2] Triyanti, V. "Risk assessment of manual material handling activities (case study: PT BRS Standard Industry)." IOP Conference Series: Materials Science and Engineering. Vol. 277. No. 1. IOP Publishing, 2017.

[3] Mohammadi, Heidar, et al. "Manual material handling assessment among workers of Iranian casting workshops." International Journal of Occupational Safety and Ergonomics19.4 (2013): 675-681.

[4] Valentina, Visentin, et al. "Fatigue accumulation in the assignment of manual material handling activities to operators." IFAC-Papers Online 51.11 (2018): 826-831.

[5] Standardised Nordic questionnaires for the analysis of musculoskeletal symptoms, I. Kuorinka, B. Jonsson t, A. Kilbom, H. Vinterberg, applied ergonomics,1987

[6] Musculoskeletal discomfort among workers in would making manufacturing industry, mohamad nasrull abdul rahmanet.al, of engineering and applied science, 2015

[7] Shojaei, Iman, et al. "Age related differences in mechanical demands imposed on the lower back by manual material handling tasks." Journal of biomechanics 49.6 (2016): 896-903.

[8] Deros, Baba Md, Dian Darina Indah Daruis, and Ishak Mohamed Basir. "A study on ergonomic awareness among workers performing manual material handling activities." Procedia-Social and Behavioral Sciences 195 (2015): 1666-1673.

[9] Parida, Ratri, and Pradip Kumar Ray. "Biomechanical modelling of manual material handling tasks: a comprehensive review." Procedia Manufacturing 3 (2015): 4598-4605.

[10] Zurada, Jozef. "Predicting the Risk of Low Back Disorders due to Manual Handling Tasks." System Science (HICSS), 2012 45th Hawaii International Conference on. IEEE, 2012. 